\title{
Use of Processed Mammography Images as a Quality Control Tool
}

\author{
Güven Bektemur ${ }^{1}$, (i) Nedim Muzoğlu², Melike Kaya Karaaslan², Mehmet Taşkın Egici ${ }^{3}$, \\ Özcan Gündoğdư 4
}

${ }^{1}$ Department of Health Management, University of Health Sciences, Istanbul, Turkey

${ }^{2}$ Department of Clinic Engineering, Istanbul Health Directorate, Istanbul, Turkey

${ }^{3}$ Department of Family Medicine, Health Sciences University, Haydarpaşa Numune Health Practice and Research Center, Istanbul, Turkey

${ }^{4}$ Department of Biomedical Engineering, Kocaeli University, Faculty of Technology, Kocaeli, Turkey

\begin{abstract}
Introduction: According to international quality control testing standards, image quality determination in mammography systems is based on measuring contrast-detail measurements with contrast-detail for mammography (CDMAM) phantom. It is therefore important to evaluate the CDMAM phantom images correctly. The purpose of this study is to compare the inverse image quality figure index $\left(\mathrm{IQF}_{\text {inv }}\right)$ obtained by automatically evaluating digital CDMAM phantom images with Artinis software (version 2.2, CDCOM) for raw and processed images.

Methods: Digital phantom images were acquired in three different full-field digital mammography systems by using automatic exposure conditions (AEC). Five different phantom images were obtained from each mammography system as raw and processed as well as evaluated by the software. In addition, the IQF ${ }_{\text {inv }}$ values of the systems were compared for mammography systems and the difference in between values was observed.

Results: The differences between the IQF $F_{\text {inv }}$ values of raw and processed images were found to be $1.34 \%, 5.62 \%$, and $6.7 \%$ for System A, B, and C, respectively.

Discussion and Conclusion: It was observed that the differences between the IQF $\mathrm{F}_{\text {inv }}$ values of raw and processed images were almost negligible and the IQF inv values obtained from the raw images were slightly higher than the values obtained from the processed images.

Keywords: CDMAM; contrast-detail; digital mammography; image quality.
\end{abstract}

$\mathrm{M}$ ammography systems are the preferred method for early detection of breast cancer not only because they are able to detect intensity changes in the breast tissue but also because they are the most cost effective and pratical methods ${ }^{[1]}$. Microcalcifications in breast tissue, depending on their size and shape, are considered to be the earliest indications of breast cancer. It is, therefore, important to obtain maximum image quality with minimal ra- diation dose in mammography systems (ALARA-principle). The performance of digital mammography systems can be evaluated by using different methods ${ }^{[2]}$. The image quality of a mammography system can be assessed objectively by contrast-detail analysis and modulation transfer function (MTF) which indicates the resolution power of the system in frequency domain ${ }^{[2]}$.

The information content of a mammographic image should

Correspondence (iletişim): Melike Kaya Karaaslan, MSc. Department of Clinic Engineering, Istanbul Health Directorate, Istanbul, Turkey Phone (Telefon): +90 5358953365 E-mail (E-posta): melikekaya08@gmail.com 
be best defined in terms of visible contrast as well as details and is characterized by a contrast-detail curve ${ }^{[3,4]}$. In this case, the minimum observable threshold contrast-detail detection is an important quality parameter for imaging systems. The contrast-detail analysis is a parameter that contains the noise, contrast, and resolution of an image as a whole. Hence, it provides a simple measurement for overall performance of the system ${ }^{[5]}$. The observable minimum distinguishable contrast detection is carried out with a low contrast test phantom that includes test objects in different sizes and contrasts.

According to European quality control testing standards and IEC 61223-3-2 (International Electrotechnical Commission), image quality measurements for digital systems are based on contrast-detail measurements with contrast-detail for mammography (CDMAM) phantom ${ }^{[3,4,6]}$. CDMAM phantom (version 3.4) is an image quality phantom designed specifically for mammography systems. CDMAM 3.4 is composed of gold disks of different thicknesses and diameters placed on an aluminum substrate. The aluminum substrate are added on a polymethyl-methacrylate (PMMA) layer. Aluminum layer and PMMA layer together are equivalent to $10 \mathrm{~mm}$ PMMA thickness. The gold disks are placed in 205 square cells in a raster fashion as 16 rows and 16 columns. The gold disks' thicknesses vary between $0.03 \mathrm{~mm}$ and $2 \mathrm{~mm}$ and their diameters vary between $0.06 \mathrm{~mm}$ and $2 \mathrm{~mm}$ as they are also logarithmically scaled. Each square is rotated by forty-five degrees to minimize the intensity difference caused by the heel effect ${ }^{[7]}$. There are two identical gold disks in each square, one at the center and one at a randomly chosen corner (of the same thickness and same diameter). The detail in the center is the reference signal and the detail in the randomly selected corner is the test signal that should be detected correctly. The ratio of correctly identified disk-positions to total number of squares, image quality figure (IQF), and inverse image quality figure $\left(\mathrm{IQF}_{\text {inv }}\right)$ are used in evaluating the image quality of CDMAM phantom images. IQF can be defined as the contrast-detail resolution of the image analysis.

In addition, IQF is considered as an adequate parameter to detect abnormal functioning of the equipment and defined in Equation $1{ }^{[1,7]}$.

$\mathrm{IQF}=\sum_{i=1}^{16} C_{i} \times D_{i, \min }(1)$

Where $\mathrm{Ci}$ is the threshold thickness of diameter $\mathrm{D}_{\mathrm{i}, \mathrm{min}}$. Image quality increases with an increasing number of correctly identified disk-positions. In this case, the IQF will become smaller because the values of diameter and thickness of the threshold-disks are smaller. The current evaluation method is to determine the threshold thickness per diameter, which is defined in Equation 2 [7].

In this method, only the number of partly visible columns of the phantom are measured and higher IQF ${ }_{\text {inv }}$ expresses better image quality ${ }^{[7]}$.

$I Q F_{\text {inv }}=\frac{100}{I Q F}(2)$

There is a linear relationship between the IQF $\mathrm{F}_{\text {inv }}$ value and the contrast-to-noise ratio (CNR) which is a preferred parameter for finding the optimal dose value. IQF $\mathrm{F}_{\text {inv }}$ has a better correlation with threshold contrast than CNR, hence $\mathrm{IQF}_{\text {inv }}$ is considered as a more reliable and objective parameter for image quality assessment ${ }^{[1,8]}$. European protocol suggests using of raw phantom images as long as they are accessible in threshold contrast detection. This is because processed images are useful for clinical diagnosis, but are useless for numerical tests, and the values obtained from raw images reflect the detector's response considerably better. There is no general standard for the definition of raw data, but in the European protocol, raw data is defined as "for processing" version of the images. However, each manufacturer applies its own device-specific "corrections" to obtain a raw image ${ }^{[3,9]}$. Moreover, raw data is not accessible for some systems, in which case, the protocol suggests that processed data can be used.

The purpose of this study is to compare the IQF ${ }_{\text {inv }}$ values of raw and processed digital phantom images.

\section{Materials and Methods}

Digital CDMAM 3.4 phantom images were taken from three different full digital mammography systems (System A, System B, and System C) with selenium (a-Se) flatpanel direct system detector technology. The detector for system $A$ had a pixel pitch of $70 \mu \mathrm{m}$ and the pixel pitch for systems B and C was $80 \mu \mathrm{m}$, manufactured in 2010, 2015, and 2010, respectively. Measurements were carried out in digital mammography systems using similar anode material. The use of a tungsten-anode tube instead of molybdenum-anode tube reduced the breast dose in the same image quality ${ }^{[10]}$. Images were obtained while System $A$ and $C$ were in Tungsten/Rhodium (W/Rh) anode/filter combination and System B in Tungsten/Silver (W/Ag) combination.

The CDMAM phantom is equivalent to $10 \mathrm{~mm}$ PMMA thickness and was placed between 4 PMMA plates of $1 \mathrm{~cm}$, with the smallest disk-diameters on the thoracic side. In order to simulate a total $60 \mathrm{~mm}$ breast thickness, images were taken using $1 \mathrm{~cm}$ spacer on the sides of $50 \mathrm{~mm}$ phantom. 
The $1 \mathrm{~cm}$ spacer was used in the test set-up because the choice of $x$-ray spectrum in many mammography systems depends on the breast thickness or the height of the compression pedal. Five different images were obtained in the automatic exposure mode (AEC) with the exposure parameters used in the clinic. The $\mathrm{IQF}_{\text {inv }}$ value is different for each image and is affected by the working conditions of the mammography system. For this reason, 5 different phantom images were acquired to obtain statistically reliable results from each system. After each image, the phantom was moved by $1 \mathrm{~mm}$ to avoid any local problem that may be present in the detector structure. Each image was recorded in DICOM format in a way that the user can access the processed data and its corresponding raw data (without pre-processing) for three systems. Artinis software (version 2.2, CDCOM) was used for the automatic evaluation of digital CDMAM phantom images. The program is able to evaluate and analyze the digital CDMAM 3.4 phantom images fast and automatically detects the location of the gold disks in the phantom image. CDCOM software is available on the EUREF website [www. euref.org].

Studies comparing $\mathrm{IQF}_{\text {inv }}$ results from raw and processed images are available in the literature ${ }^{[11-14]}$, but it is recommended that $1 \mathrm{~cm}$ spacer should be used on phantom edges to better simulate breast thickness with respect to European standards. For this reason, in our study, $60 \mathrm{~mm}$ breast thickness was simulated by using $1 \mathrm{~cm}$ thick spacer.

\section{Results}

Raw and processed phantom images were acquired from three different digital mammography systems and then $\mathrm{IQF}_{\text {inv }}$ values were obtained by evaluating with the Artinis software (V.2.2).

$\mathrm{IQF}_{\text {inv }}$ values for System $A, B$, and $C$ are shown for raw and processed images and the difference between them is expressed as percentage (\%) in Table 1.

The difference between the $\mathrm{IQF}_{\text {inv }}$ values were $1.34 \%$, $5.62 \%$, and $6.7 \%$ for System A, B, and C, respectively. The difference of IQF ${ }_{\text {inv }}$ values between raw and processed im-

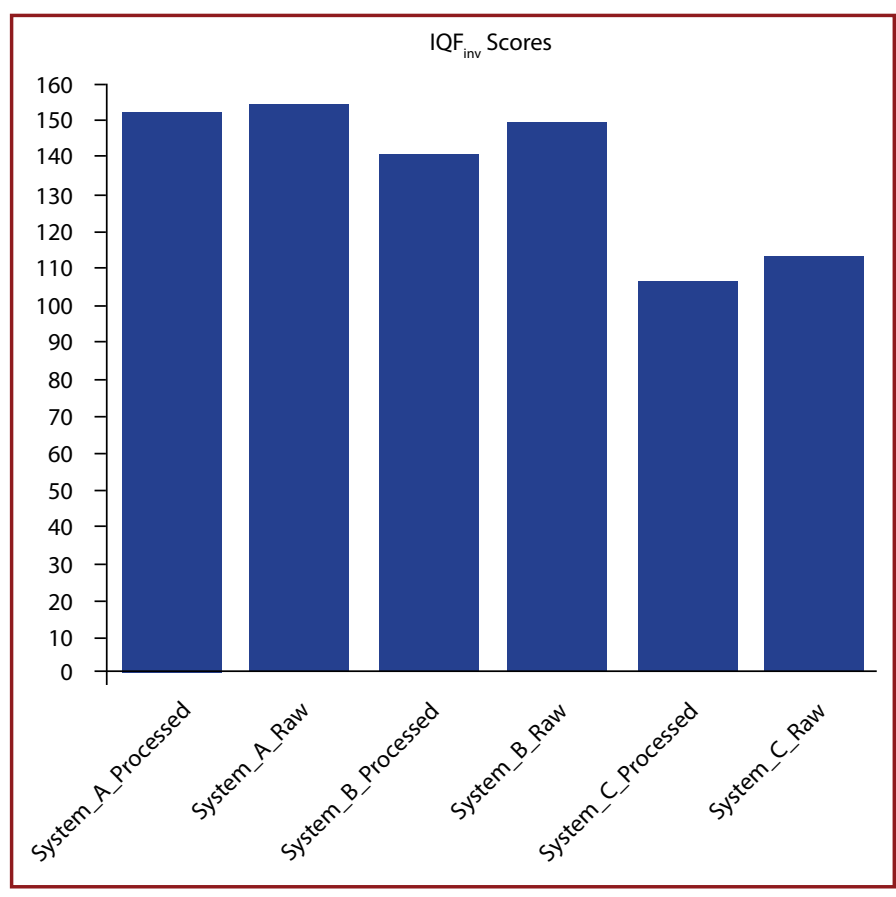

Figure 1. Comparison of Inverse image quality figure index $\left(\mathrm{IQF}_{\mathrm{inv}}\right)$ values of systems for raw and processed images.

ages differs from system to system as manufacturers employ different image processing algorithms such as $\mathrm{Mu}$ sica 1, IMS Raffaello, OpView v1, OpView v2, and Sigmoid in the processing of images. The IQF $F_{\text {inv }}$ values of the raw and processed images for System A, B, and C are shown graphically in Figure 1.

\section{Conclusions}

European standards recommend the use of raw images for the detection of the contrast-detail curve in previous studies. However, it has been observed that there is no significant difference between the IQF $\mathrm{F}_{\text {inv }}$ values of raw and processed images calculated by the software. The difference between the IQF ${ }_{\text {inv }}$ values obtained from raw and processed images differs from system to system, so it is not possible to generalize this conclusion for all digital mammography systems. In addition, when IQF ${ }_{\text {inv }}$ values are used to compare different systems, similar results are observed for raw and processed images.

Table 1. Inverse image quality figure index $\left(\mathrm{IQF}_{\text {inv }}\right)$ values of raw and processed images

\begin{tabular}{lccc}
\hline $\begin{array}{l}\text { Mammography } \\
\text { systems }\end{array}$ & Raw_IQF $_{\text {inv }}$ & Processed_IQF $_{\text {inv }}$ & $\begin{array}{c}\text { \% Difference two } \\
\text { groups between }\end{array}$ \\
\hline A & 154.38 & 152.33 & 1.34 \\
B & 148.61 & 140.69 & 5.62 \\
C & 113.05 & 105.95 & 6.7 \\
\hline
\end{tabular}


Peer-review: Externally peer-reviewed.

Authorship Contributions: Concept: G.B., N.M., M.K.K., M.T.E., Ö.G.; Design: G.B., N.M., M.K.K., M.T.E., Ö.G.; Data Collection or Processing: G.B., N.M., M.K.K., M.T.E., Ö.G.; Analysis or Interpretation: G.B., N.M., M.K.K., M.T.E., Ö.G.; Literature Search: G.B., N.M., M.K.K., M.T.E., Ö.G.; Writing: G.B., N.M., M.K.K., M.T.E., Ö.G.

Conflict of Interest: None declared.

Financial Disclosure: The authors declared that this study received no financial support.

\section{References}

1. Mayo P, Rodenas F, Verdú G, Campayo JM. Analysis of Digital image quality indexes for CIRS SP01 and CDMAM 3.4 mammographic phantoms. In: Dössel O, Schlegel WC, editors. World Congress on Medical Physics and Biomedical Engineering; 2009 Sept 7 - 12; Munich, Germany. [CrossRef]

2. Young KC, Oduko JM, Gundogdu O, Alsager A. Comparing the Performance of Digital Mammography Systems. In: Krupinski EA, editor. Digital Mammography. Lecture Notes in Computer Science. Vol 5116. Berlin: Springer; 2008. p. 732-39. [CrossRef]

3. European Reference Organisation for Quality Assured Breast Screening and Diagnostic Services. Protocol for the Quality Control of the Physical and Technical Aspects of Mammography Screening. European Commission Netherlands: European Commission; 2014.

4. Health \& Consumer Protection Directorate-General. European guidelines for quality assurance in breast cancer screening and diagnosis. 4th ed. European Commission; 2006.

5. Lyra ME, Kordolaimi SD, Salvara ALN. Presentation of Digital Radiographic Systems and the Quality Control Procedures that Currently Followed by Various Organizations Worldwide. Recent Patents on Medical Imaging 2010;2:5-21. [CrossRef]

6. IEC 61223-3-2:2007 Evaluation and routine testing in medical imaging departments - Part 3-2: Acceptance tests - Imaging performance of mammographic X-ray equipment. Geneva:
International Electrotechnical Commission; 2007.

7. Floor M, Van Der Burght R. Manuel Contrast-Detail Phantom CDMAM 3.4 and CDMAM 3.4 Analyser Software V2.2. Netherlands; Artinis Medical System; 2014.

8. Oberhofer N, Paruccini N, Moroder E. Image Quality Assessment and Equipment Optimisation with Automated Phantom Evaluation in Full Field Digital Mammography (FFDM). In: Krupinski EA, editor. Lecture Notes in Computer Science. Vol 5116. Berlin: Springer; 2008. p. 235-42. [CrossRef]

9. Bacher K. Evaluation of image quality and patient radiation dose in digital radiology [Dissertation]. Gent: Gent University; 2006.

10. Oduko JM, Young KC, Gundogdu O, Alsager A. Effect of Using Tungsten-Anode X-Ray Tubes on Dose and Image Quality in Full-Field Digital Mammography. Lecture Notes in Computer Science. Vol 5116. Berlin: Springer; 2008. p. 522-9. [CrossRef]

11. Ruben E, Van E, Young C.K, Bosmans H, Lazzari B, Schopphoven S, Heid P, Thijssen M. A supplement to the European Guidelines for Quality Assurance in Breast Cancer Screening and Diagnosis. In: Martí J, Oliver A, Freixenet J, Martí R, editors. Lecture Notes in Computer Science. Vol 6136. Berlin: Springer.

12. Zanca F, Bosmans H, Jacobs J, Michielsen K, Sisini F, Nens J, et al. Contrast-Detail comparison between unprocessed and processed CDMAM images. Proceedings of the SPIE 7258, Medical Imaging: Physics of Medical Imaging; 2009 Mar 14. Lake Buena Vista, USA: SPIE; 2009. [CrossRef]

13. Collado-Chamorro $P$, Sanz-Freire $C$, Gómez-Amez J, VázquezGaliñanes A, Lopo-Casqueiro N, González-De La Puente M. Contrast Detail Curves on Digital Mammography: Performance Comparison of Raw and Filtered Images. In: Martí J, Oliver A, Freixenet J, Martí R, editors. Lecture Notes in Computer Science. Vol 6136. Berlin: Springer; 2010. p. 304-11.

14. Borg M, Badr I, Royle GJ. Should Processed or Raw image Data Be Used in Mammographic Image Quality analyses? A Comparative Study of Three Full-Field Digital Mammography Systems. Radiation Protection Dosimetry 2010;163:102-17. 